Research Article

\title{
Output Monomial Reachability and Zero Output Controllability of Positive Switched Systems
}

\author{
Maryam Riouali $\left(\mathbb{D},{ }^{1}\right.$ Fouad Lahmidi, ${ }^{1}$ and Abdelwahed Namir ${ }^{2}$ \\ ${ }^{1}$ Laboratory of Analysis, Modeling and Simulation, Faculty of Sciences Ben M'sik, Hassan II University, B.P 7955, \\ Casablanca, Morocco \\ ${ }^{2}$ Laboratory of Information Technology and Modeling, Faculty of Sciences Ben M'sik, Hassan II University, B.P 7955, \\ Casablanca, Morocco \\ Correspondence should be addressed to Maryam Riouali; maryam.riouali1@gmail.com
}

Received 6 May 2020; Revised 23 August 2020; Accepted 21 September 2020; Published 15 October 2020

Academic Editor: Hans Engler

Copyright () 2020 Maryam Riouali et al. This is an open access article distributed under the Creative Commons Attribution License, which permits unrestricted use, distribution, and reproduction in any medium, provided the original work is properly cited.

In this paper, we present a sufficient condition for the output reachability of discrete-time positive switched systems. Besides, necessary and sufficient conditions for output monomial reachability and zero output controllability are provided. Further, some examples are shown.

\section{Introduction}

In recent years, engineers and applied mathematicians have been interested in the study and analysis of switched systems, which represent an important class of hybrid dynamical systems. A switched system is the association of a finite set of differential or difference subsystems and a switching law that indicates at each instant the active system. Switched systems are of a great interest, since they are very convenient in the mathematical modelling of several systems such as network control systems, near-space vehicle control systems [1], biological systems [2], dc/dc convertors, oscillators [3], chaos generators [4], and so on. Based on previous research, many mathematical problems have been posed and investigated such as stability and stabilizability properties $[5,6]$. Recent studies examined other issues such as reachability and controllability [7-13]. It is important to note that Babiarz [14] provided important results on the output controllability for standard switched systems.

It should be noted that positive systems are of great importance in practice as they appear naturally in various fields of science and technology. They have the property that all descriptive variables can only take positive values, or at least nonnegative values. These systems can be found in economics [15], biology, stochastic processes (Markov chains or hidden Markov models) [16], chemical processing [17], communication science and information [18], etc. The theory of positive systems is more complicated than the one of the standard systems because positive linear systems are defined on cones and not on linear spaces [19]. As a result, some known properties of linear systems cannot be applied for positive systems (for more details, see [20]).

Combing the characteristics of general switched systems and positive systems, results are obtained on positive switched systems [21]. The strong interest that this type of system has recently raised is due to its strong presence in the most important areas. As an example, in the field of biology and pharmacokinetics, they are used to describe the dynamics of the viral mutation under drug treatment [22]. It is also applied in HIV treatment modelling [21], formation flying [23], and communication systems [18]. Many problems have been examined concerning positive switched systems, such as stability and stabilizability [24] as well as structural properties, like reachability, controllability, and observability $[25,26]$.

This paper which deals with the output reachability and controllability problem of discrete-time positive switched systems is organized as follows. After some preliminaries in 
the following section, we provide necessary conditions for the output reachability in Section 3. In Section 4, necessary and sufficient conditions for the output monomial reachability are provided. The zero output controllability problem is explored in Section 5.

\section{Preliminaries}

The symbols $\mathbb{Z}_{+}$and $\mathbb{R}_{+}$denote the sets of nonnegative integers and nonnegative real numbers, respectively. $\mathbb{R}^{n}$ is the $n$-dimensional Euclidean space and $\mathbb{R}_{+}^{n}$ is the set of all $\mathrm{n}$-dimensional nonnegative real vectors. In addition, $\mathbb{R}^{n \times m}$ represents the space of $n \times m$ matrices with real entries and $\mathbb{R}_{+}^{n \times m}$ represents the set of all $n \times m$ matrices with nonnegative entries. If $E \in \mathbb{R}_{+}^{n \times m}$, we say that $E$ is nonnegative and write $E \geq 0$. We write $A^{T}$ for the transpose of the matrix $A$ and $I_{n}$ the $n \times n$ identity matrix. For any $i, j \in \mathbb{Z}_{+}$, with $i \leq j$, we set $[i, j]=\{i, i+1, \ldots, j\}$.

Let $\mathscr{A}=\left\{a_{1}, a_{2}, \ldots, a_{r}\right\}, r \in \mathbb{Z}_{+} \backslash\{0\}$, be an alphabet whose elements are called letters. A word over the alphabet $\mathscr{A}$ is a finite sequence of elements of $\mathscr{A}$; it will be denoted by $\omega=a_{i_{1}} a_{i_{2}}, \ldots, a_{i_{l}}$ where $a_{i_{j}} \in \mathscr{A}, l \in \mathbb{Z}_{+} \backslash\{0\}$. The length of the word $\omega$ is the number of letters it is composed of, written as $|\omega|=l$.

The set of all words over the alphabet is a free monoid $\mathscr{A}^{*}$ for concatenation, whose neutral element is the empty word denoted by $\varepsilon=\varnothing$. Clearly, for any word $\omega \in \mathscr{A}^{*}, \varepsilon \omega=\omega \varepsilon=$ $\omega$ and $|\varepsilon|=0$.

Let $\left\{A_{1}, A_{2}, \ldots, A_{r}\right\}$ be a set of $n \times n$ matrices and $i_{1}, i_{2}, \ldots, i_{l} \in[1, r]$. If $\omega$ is a word in $\mathscr{A}^{*}$, we set

$\omega\left(A_{1}, A_{2}, \ldots, A_{r}\right)= \begin{cases}I_{n}, & \text { if } \omega=\varepsilon, \\ A_{i_{1}} A_{i_{2}}, \ldots, A_{i_{l}}, & \text { if } \omega=a_{i_{1}} a_{i_{2}}, \ldots, a_{i_{l}} .\end{cases}$

Next, we introduce a class of nonnegative matrices, namely, the monomial matrices.

Definition 1. A nonnegative vector $v$ is said to be monomial if it contains precisely one nonzero entry. We will call it an $i$-monomial vector if the nonzero component is in the $i$ th position.

Definition 2. A nonnegative matrix $E$ is a monomial matrix if it has only one nonzero entry in every row and every column.

In this paper, we consider a discrete-time switched system described by the difference state equation

$$
\left\{\begin{array}{l}
x_{t+1}=A_{\delta(t)} x_{t}+B_{\delta(t)} u_{t}, \quad t \in \mathbb{Z}_{+}, \\
y_{t}=C_{\delta(t)} x_{t}
\end{array}\right.
$$

where $x_{t} \in \mathbb{R}^{n}$ is the state vector, $u_{t} \in \mathbb{R}^{m}$ is the control input, $y_{t} \in \mathbb{R}^{p}$ is the output vector, and $\delta: \mathbb{Z}_{+} \longrightarrow \Omega=[1, r]$ is a switching sequence.

Given a control $u_{t}, t \in[0, k-1]$, and a switching sequence $\delta(t), t \in[0, k-1]$, the solution of system (2), with the initial condition $x_{0}$, at time $k$, can be expressed as [25]

$$
x_{k}=\mathscr{H}_{\delta}(k, 0) x_{0}+\sum_{t=0}^{k-1} \mathscr{H}_{\delta}(k, t+1) B_{\delta(t)} u_{t} \text {, }
$$

where

$$
\mathscr{H}_{\delta}(t, l)=\left\{\begin{array}{lllll}
I_{n}, & & & t=l \geq 0, \\
A_{\delta(t-1)} & A_{\delta(t-2)} & \cdots & A_{\delta(l)} & t>l .
\end{array}\right.
$$

Definition 3. The discrete system (2) is called positive if for any switching sequence $\delta$, any initial condition $x_{0} \in \mathbb{R}_{+}^{n}$, and for any input $u_{t} \in \mathbb{R}_{+}^{m}, t \in \mathbb{Z}_{+}$, the state $x_{t} \in \mathbb{R}_{+}^{n}$ and the output $y_{t} \in \mathbb{R}_{+}^{p}$ for all $t \in \mathbb{Z}_{+}$.

Proposition 1. The discrete system (2) is positive if and only if, for each $i \in \Omega, A_{i} \in \mathbb{R}_{+}^{n \times n}, B_{i} \in \mathbb{R}_{+}^{n \times m}$, and $C_{i} \in \mathbb{R}_{+}^{p \times n}$.

Proof. If $A_{i} \in \mathbb{R}_{+}^{n \times n}, B_{i} \in \mathbb{R}_{+}^{n \times m}$, and $C_{i} \in \mathbb{R}_{+}^{p \times n}$ for all $i \in \Omega$, then equations (3) and (4) imply that for all $x_{0} \in \mathbb{R}_{+}^{n}$ and $u_{t} \in \mathbb{R}_{+}^{m}, t \in \mathbb{Z}_{+}$we have $x_{t} \in \mathbb{R}_{+}^{n}$ and $y_{t} \in \mathbb{R}_{+}^{p}$ for all $t \in \mathbb{Z}_{+}$.

Conversely, assume that the positive switched system (1) is positive. Let $\delta(t)=i, i \in \Omega$, and $u_{t}=0$ for all $t \in \mathbb{Z}_{+}$. Then, from (2), for $t=0$, we obtain $x_{1}=A_{i} x_{0} \in \mathbb{R}_{+}^{n}$ and $y_{0}=C_{i} x_{0} \in \mathbb{R}_{+}^{p}$. Thus, $A_{i} \in \mathbb{R}_{+}^{n \times n}$ and $C_{i} \in \mathbb{R}_{+}^{p \times n}$, since $x_{0}$ may be arbitrary. Now, assuming that $x_{0}=0$, then from (2), for $t=0$, we have $x_{1}=B_{i} u_{0} \in \mathbb{R}_{+}^{n}$. It follows that $B_{i} \in \mathbb{R}_{+}^{n \times m}$, since $u_{0}$ may be arbitrary.

\section{Output Reachability of Switched Positive Systems}

In the main result of this section, we provide a sufficient condition for the output reachability of system (2). Before giving our result, some definitions concerning the output reachability of positive switched systems should be cited.

Definition 4. An output $y_{d} \in \mathbb{R}_{+}^{p}$ is said to be reachable in $N$ steps if there exists a switching sequence $\delta(t), t \in[0, N]$, and inputs $u_{t} \in \mathbb{R}_{+}^{m}$ for $t \in[0, N-1]$ that steer the output of system (2) from $x_{0}=0$ to $y_{d}$, namely, $y_{d}=y_{N}$. System (2) is called output reachable if every nonnegative output $y_{d} \in \mathbb{R}_{+}^{p}$ is reachable in some step $N$.

It is clearly seen that when $x_{0}=0$, the output can be written as

$$
y_{k}=\mathscr{R}_{k}(\delta) u^{k}
$$

where

$$
\begin{aligned}
\mathscr{R}_{k}(\delta) & =\left[C_{\delta(k)} \mathscr{H}_{\delta}(k, 1) B_{\delta(0)} C_{\delta(k)} \mathscr{H}_{\delta}(k, 2) B_{\delta(1)} \cdots C_{\delta(k)} B_{\delta(k-1)}\right], \\
u^{k} & =\left[\begin{array}{c}
u_{0} \\
u_{1} \\
\vdots \\
u_{k-1}
\end{array}\right] .
\end{aligned}
$$


$\mathscr{R}_{k}(\delta)$ is called the output reachability matrix associated to the switching sequence $\delta$.

Definition 5. The set of all nonnegative linear combinations of the columns of a matrix $A \in \mathbb{R}^{p \times n}$ is called polyhedral convex cone, namely,

$$
\text { Cone }(A)=\left\{y \in \mathbb{R}^{p}: y=A x, x \in \mathbb{R}_{+}^{n}\right\} .
$$

Polyhedral convex cones play an important role in the output reachability of positive systems since the set of all reachable outputs in $k$ steps is a polyhedral cone belonging to the nonnegative orthant.

Cone $\left(\mathscr{R}_{k}(\delta)\right)$ is a polyhedral cone generated by the columns of the output reachability matrix $\mathscr{R}_{k}(\delta)$ associated to the switching sequence $\delta$ of length $k+1$. The length of the switching sequence $\delta$ is the cardinality of the discrete-time interval $[0, k]$ and it is denoted, for short, by means of the notation $|\delta|=k+1$.

Clearly, the positive switched system (2) is output reachable if there exist switching sequences $\delta_{1}, \delta_{2}, \ldots, \delta_{h}$ of lengths $k_{1}+1, k_{2}+1, \ldots, k_{h}+1$, respectively, $h \geq 1$, such that

$$
\bigcup_{t=1}^{h} \operatorname{Cone}\left(\mathscr{R}_{k_{t}}\left(\delta_{t}\right)\right)=\mathbb{R}_{+}^{p} \text {. }
$$

Example 1. Consider positive switched system (2) with $\Omega=$ $[1,2]$ and the following matrices:

$$
\begin{aligned}
& A_{1}=\left(\begin{array}{ll}
1 & 1 \\
1 & 1
\end{array}\right), \\
& A_{2}=\left(\begin{array}{ll}
1 & 1 \\
0 & 0
\end{array}\right), \\
& B_{1}=\left(\begin{array}{ll}
0 & 1 \\
1 & 0
\end{array}\right), \\
& B_{2}=\left(\begin{array}{ll}
1 & 0 \\
0 & 1
\end{array}\right), \\
& C_{1}=\left(\begin{array}{ll}
1 & 1 \\
0 & 1
\end{array}\right), \\
& C_{2}=\left(\begin{array}{ll}
1 & 0 \\
1 & 1
\end{array}\right) .
\end{aligned}
$$

Define

$$
\begin{aligned}
& \left\{\begin{array}{l}
\delta_{1}(0)=2, \\
\delta_{1}(1)=2,
\end{array}\right. \\
& \left\{\begin{array}{l}
\delta_{2}(0)=1, \\
\delta_{2}(1)=1 .
\end{array}\right.
\end{aligned}
$$

We get

$$
\begin{aligned}
& \text { Cone }\left(\mathscr{R}_{1}\left(\delta_{1}\right)\right) \cup \operatorname{Cone}\left(\mathscr{R}_{1}\left(\delta_{2}\right)\right) \\
& =\text { Cone }\left(\begin{array}{ll}
1 & 0 \\
1 & 1
\end{array}\right) \cup \text { Cone }\left(\begin{array}{ll}
1 & 1 \\
1 & 0
\end{array}\right)=\mathbb{R}_{+}^{2} .
\end{aligned}
$$

Therefore, the system is output reachable.

\section{Output Monomial Reachability of Switched Positive Systems}

We study in this section the concept of output monomial reachability and provide necessary and sufficient conditions for this property. First, we recall the following definition and give some preliminary results.

Definition 6. The positive switched system (2) with $x_{0}=0$ is said to be output monomially reachable if, for all $i \in[1, p]$, there exist $k \geq 1$, a switching sequence $\delta:[0, k] \longrightarrow \Omega$, and nonnegative control inputs $u_{0}, u_{1}, \ldots, u_{k-1}$ such that

$$
y_{k}=e_{i} \text {, }
$$

with $e_{i}$ being the $i$ th canonical vector of $\mathbb{R}^{p}$.

Lemma 1. If $B \in \mathbb{R}_{+}^{m \times n}$ and $V \in \mathbb{R}_{+}^{n}$ are such that $B V$ is an $i$ monomial vector, then $B$ includes an i-monomial column.

Proof. Let $B=\left(\begin{array}{llll}b_{1} & b_{2} & \ldots & b_{n}\end{array}\right) \in \mathbb{R}_{+}^{m \times n}$, with $b_{l}=\left(b_{1 l}\right.$, $\left.b_{2 l}, \ldots, b_{m l}\right)^{T}$ being the vector columns of $B$ and $V=\left(v_{1}, v_{2}, \ldots, v_{n}\right)^{T} \in \mathbb{R}_{+}^{n}$. If $B V$ is $i$-monomial, then

$$
B V=v_{1} b_{1}+v_{2} b_{2}+\cdots+v_{n} b_{n}=(0,0, \ldots, \alpha, 0, \ldots, 0)^{T},
$$

with $\alpha>0$,

which implies that for all $j \in[1, n]$, we have $v_{j} b_{j}=$ $\left(0,0, \ldots, v_{j} b_{i j}, 0, \ldots, 0\right)^{T}$ and there exists some $j \in[1, n]$ such that $v_{j} b_{j} \neq 0$. Therefore, there exists $j \in[1, n]$ such that $b_{j}=\left(0,0, \ldots, b_{i j}, 0, \ldots, 0\right)^{T} \neq 0$. Hence, $B$ includes an $i$ monomial column.

Corollary 1. Let $A \in \mathbb{R}_{+}^{p \times n}$ and $B \in \mathbb{R}_{+}^{n \times m}$. If $A B$ includes an $i$-monomial column, then $A$ has an i-monomial column.

Proof. Let $b_{1}, b_{2}, \ldots, b_{m}$ be the vector columns of $B$; then,

$$
A B=\left(\begin{array}{llll}
A b_{1} & A b_{2} & \ldots & A b_{m}
\end{array}\right)
$$

Since $A B$ contains an $i$-monomial column, then there exists $j \in[1, m]$ such that $A b_{j}$ is an $i$-monomial vector. Applying Lemma 1, it yields that the matrix $A$ has an $i$ monomial vector.

The proposition below contains a necessary and sufficient condition for output monomial reachability using the output reachable matrix $\mathscr{R}_{N}$ associated with all possible switching sequences.

Proposition 2. The positive switched system (2) is output monomially reachable if and only if there exists some positive integer $N$ such that the output reachability matrix in $N$ steps 


$$
\begin{array}{r}
\mathscr{R}_{N}=\left[C_{1} \omega\left(A_{1}, \ldots, A_{r}\right) B_{1} \cdots C_{1} \omega\left(A_{1}, \ldots, A_{r}\right) B_{r} C_{2} \omega\left(A_{1}, \ldots, A_{r}\right) B_{1} \ldots C_{r} \omega\left(A_{1}, \ldots, A_{r}\right) B_{r}\right] \omega \in \mathscr{A}^{*}, \\
0 \leq|\omega| \leq N-1
\end{array}
$$

includes an $p \times p$ monomial submatrix.

Proof. Assume that for all $i \in[1, p]$, there exist $k \geq 1$, a switching sequence $\delta:[0, k] \longrightarrow \Omega$, and nonnegative control inputs $u_{0}, u_{1}, \ldots, u_{k-1}$ such that $y_{k}=e_{i}$. This implies that the following equality $\sum_{t=0}^{k-1} C_{\delta(k)} \mathscr{H}_{\delta}(k, t+1) B_{\delta(t)}$ $u_{t}=e_{i}$ holds.

Then, there exists $l \in[1, k]$ such that $\left(C_{\delta(k)} A_{\delta(k-1)} \cdots A_{\delta(l)} B_{\delta(l-1)} u_{l-1}\right)$ is an $i$-monomial vector. By Lemma 1, $\left(C_{\delta(k)} A_{\delta(k-1)} \cdots A_{\delta(l)} B_{\delta(l-1)}\right)$ includes an $i$ monomial column. Let

$$
\omega_{i}=\left\{\begin{array}{lllll}
\varepsilon, & & & & \text { if } k=l, \\
a_{\delta(k-1)} & a_{\delta(k-2)} & \cdots & a_{\delta(l)}, & \text { if } l \leq k-1,
\end{array}\right.
$$

and pose $\delta(k)=l_{i}$ and $\delta(l-1)=j_{i}$. Then, $C_{l_{i}} \omega_{i}$ $\left(A_{1}, A_{2}, \ldots, A_{r}\right) B_{j_{i}}$ includes an $i$-monomial column. For $N=\max _{1 \leq i \leq p}\left|\omega_{i}\right|+1$, we have $\mathscr{R}_{N}$ includes an $p \times p$ monomial submatrix.

Conversely, let $i \in[1, p]$; then, $\mathscr{R}_{N}$ includes an $i$-monomial column, which implies that there exist $\omega_{i} \in \mathscr{A}^{*}$, $0 \leq\left|\omega_{i}\right| \leq N-1$, and $l_{i}, j_{i} \in \Omega$ such that $C_{l_{i}} \omega_{i}\left(A_{1}\right.$, $\left.A_{2}, \ldots, A_{r}\right) B_{j_{i}}$ contains an $i$-monomial column.

Let $k=\left|\omega_{i}\right|+1$ and pose

$$
\omega_{i}= \begin{cases}\varepsilon, & \text { if }\left|\omega_{i}\right|=0 \\ a_{i_{k-1}} a_{i_{k-2}}, \ldots, a_{i_{1}}, & \text { if }\left|\omega_{i}\right| \geq 1\end{cases}
$$

Let $\delta:[0, k] \longrightarrow \Omega$ satisfying $\delta(0)=j_{i}, \delta(k)=l_{i}$ and $\delta(h)=i_{h}$, where $1 \leq h \leq k-1$. Then,

$$
C_{l_{i}} \omega_{i}\left(A_{1}, A_{2}, \ldots, A_{r}\right) B_{j_{i}}=\left(C_{\delta(k)} A_{\delta(k-1)} \cdots A_{\delta(1)} B_{\delta(0)}\right) .
$$

Set $M=\left(C_{\delta(k)} A_{\delta(k-1)} \cdots A_{\delta(1)} B_{\delta(0)}\right)$. Then, there exists $j \in[1, m]$ such that $M_{i j}>0$ and $M_{l j}=0$, for $l \in[1, p] \backslash\{i\}$.

Let $u_{0}=\left(0, \ldots, 0,\left(1 / M_{i j}\right), 0, \ldots, 0\right)^{T} \in \mathbb{R}_{+}^{m}$ and $u_{l}=$ $0 \in \mathbb{R}_{+}^{m}$, for all $j \in[1, k-1]$.

We get from (5) that

$$
y_{k}=\left(C_{\delta(k)} A_{\delta(k-1)} \cdots A_{\delta(1)} B_{\delta(0)} u_{0}\right)=M u_{0}=e_{i} \text {. }
$$

Therefore, the system is output monomially reachable.

Remark 1. In the case of single output systems $(p=1)$, the Proposition 2 gives in fact a characterization of the output reachability of system (2).

Let us now consider some examples.

Example 2. Consider positive switched system (2) consisting of two subsystems with the following matrices:

$$
\begin{aligned}
& \left(A_{1}, B_{1}, C_{1}\right)=\left(\left[\begin{array}{ll}
1 & 0 \\
1 & 0
\end{array}\right],\left[\begin{array}{l}
1 \\
0
\end{array}\right],\left[\begin{array}{ll}
0 & 1
\end{array}\right]\right), \\
& \left(A_{2}, B_{2}, C_{2}\right)=\left(\left[\begin{array}{ll}
0 & 1 \\
0 & 1
\end{array}\right],\left[\begin{array}{l}
0 \\
1
\end{array}\right],\left[\begin{array}{ll}
1 & 0
\end{array}\right]\right) .
\end{aligned}
$$

For the two subsystems, we have $x_{0}=0, y_{1}=C_{i} B_{i} u_{0}=0$, and $i \in[1,2]$. So, neither one is output reachable in one step. But $\mathscr{R}_{1}=\left[C_{1} B_{1} C_{1} B_{2} C_{2} B_{1} C_{2} B_{2}\right]=\left[\begin{array}{llll}0 & 1 & 1 & 0\end{array}\right]$, and hence the positive system (2) is output reachable in one step. Indeed, let $\delta(0)=1$ and $\delta(1)=2$; then, for all $y_{d} \in \mathbb{R}_{+}$, for $u_{0}=y_{d}$ we get $y_{1}=C_{2} B_{1} u_{0}=y_{d}$.

Example 3. Consider the positive system switching among the following subsystems:

$$
\begin{aligned}
& \left(A_{1}, B_{1}, C_{1}\right)=\left(\left[\begin{array}{ll}
1 & 0 \\
1 & 0
\end{array}\right],\left[\begin{array}{l}
1 \\
0
\end{array}\right],\left[\begin{array}{ll}
0 & 0 \\
1 & 0
\end{array}\right]\right), \\
& \left(A_{2}, B_{2}, C_{2}\right)=\left(\left[\begin{array}{ll}
0 & 1 \\
0 & 1
\end{array}\right],\left[\begin{array}{l}
0 \\
1
\end{array}\right],\left[\begin{array}{ll}
0 & 1 \\
0 & 0
\end{array}\right]\right) .
\end{aligned}
$$

We have

$$
\begin{aligned}
& C_{1} B_{1}=\left[\begin{array}{l}
0 \\
1
\end{array}\right], \\
& C_{2} B_{2}=\left[\begin{array}{l}
1 \\
0
\end{array}\right] .
\end{aligned}
$$

So, the two subsystems are not output monomially reachable in one step. But

$$
R_{1}=\left[\begin{array}{llll}
C_{1} B_{1} & C_{1} B_{2} & C_{2} B_{1} & C_{2} B_{2}
\end{array}\right]=\left[\begin{array}{llll}
0 & 0 & 0 & 1 \\
1 & 0 & 0 & 0
\end{array}\right] .
$$

Hence, the positive system (2) is output monomially reachable in one step. Indeed, for any $y_{d}=\left[\begin{array}{l}\alpha \\ 0\end{array}\right]$, let $\delta(0)=$ $\delta(1)=2$ and $u_{0}=\alpha$. Then, $y_{1}=C_{2} B_{2} u_{0}=\left[\begin{array}{l}\alpha \\ 0\end{array}\right]=y_{d}$.

Also, for any $y_{d}=\left[\begin{array}{l}0 \\ \beta\end{array}\right]$, let $\delta(0)=\delta(1)=1$ and $u_{0}=\beta$. Then, $y_{1}=C_{1} B_{1} u_{0}=\left[\begin{array}{l}0 \\ \beta\end{array}\right]=y_{d}$.

On the other hand, it is clearly seen that this system is not reachable in one step because the vector $\left[\begin{array}{l}1 \\ 1\end{array}\right]$ can never be reached in one step.

Corollary 2. If the positive switched system (2) is output monomially reachable, then the matrix $\left[\begin{array}{llll}C_{1} & C_{2} & \cdots & C_{r}\end{array}\right]$ has an $p \times p$ monomial submatrix. 
Proof. Suppose the system is output monomially reachable. Thus, for all $i \in[1, p]$, there exist $\omega_{i} \in \mathscr{A}^{*}, j_{i}, l_{i} \in \Omega$ such that $C_{l_{i}} \omega_{i}\left(A_{1}, A_{2}, \ldots, A_{r}\right) B_{j_{i}}$ has an $i$-monomial column. Applying Corollary 1 , it yields that the matrix $C_{l_{i}}$ has an $i$ monomial column.

Hence, the matrix $\left[\begin{array}{llll}C_{1} & C_{2} & \cdots & C_{r}\end{array}\right]$ has an $p \times p$ monomial submatrix.

\section{Zero Output Controllability}

To present our main results for zero output controllability, we introduce the following definition.

Definition 7. The positive switched system (2) is said to be zero output controllable if, for all $x_{0} \in \mathbb{R}_{+}^{n}$, there exist $k \geq 1$, a switching sequence $\delta:[0, k] \longrightarrow \Omega$, and nonnegative control input $u_{t} \in \mathbb{R}_{+}^{m}, t \in[0, k-1]$ such that

$$
y_{k}=0 \text {. }
$$

Proposition 3. The positive switched system (2) is zero output controllable if and only if there exist $\omega \in \mathscr{A}^{*}$ and $j \in[1, r]$ such that

$$
C_{j} \omega\left(A_{1}, A_{2}, \ldots, A_{r}\right)=0 .
$$

Proof. If the system is zero output controllable, then in particular, for $x_{0}=(1,1, \ldots, 1)^{T}$, there exist $k \geq 1$, a switching sequence $\delta:[0, k] \longrightarrow \Omega$, and $u_{t} \in \mathbb{R}_{+}^{m}, t \in[0, k-$ 1] such that $y_{k}=0$.

It follows that

$$
\left(C_{\delta(k)} A_{\delta(k-1)} \cdots A_{\delta(0)} x_{0}\right)=0 .
$$

Then,

$$
\left(C_{\delta(k)} A_{\delta(k-1)} \cdots A_{\delta(0)}\right)=0 .
$$

Let $\omega=\left(a_{\delta(k-1)} \cdots a_{\delta(0)}\right)$ and $j=\delta(k)$; then, $C_{j} \omega\left(A_{1}, A_{2}, \ldots, A_{r}\right)=0$.

Conversely, let $k=|\omega|$ with $\omega=\left(a_{i_{k-1}} \cdots a_{i_{0}}\right), \delta(l)=i_{l}$, $l \in[0, k-1], \delta(k)=j$, and $u_{0}=\cdots=u_{k-1}=0$. Then, for each $x_{0} \in \mathbb{R}_{+}^{n}$, we have

$$
y_{k}=\left(C_{\delta(k)} A_{\delta(k-1)} \cdots A_{\delta(0)} x_{0}\right)=C_{j} \omega\left(A_{1}, A_{2}, \ldots, A_{r}\right) x_{0}=0 .
$$

If $|\omega|=0$, then $C_{j}=0$, and for $\delta(0)=\delta(1)=j$ and $u_{0}=0$, we obtain $y_{1}=0$, for all $x_{0} \in \mathbb{R}_{+}^{n}$, which completes the proof.

Corollary 3. The positive switched system (2) is zero output controllable if there exists $\omega \in \mathscr{A}^{*}$ such that $\omega\left(A_{1}\right.$, $\left.A_{2}, \ldots, A_{r}\right)$ is nilpotent.

Proof. Assume that there exists $\omega=\left(a_{i_{1}} \cdots a_{i_{k}}\right)$ such that $\left(\omega\left(A_{1}, A_{2}, \ldots, A_{r}\right)\right)^{N}=\left[A_{i_{1}} \cdots A_{i_{k}} A_{i_{1}} \cdots A_{i_{k}} \cdots \cdots A_{i_{1}} \cdots A_{i_{k}}\right]=0$.

Let $\quad \tilde{\omega}=\left[a_{i_{1}} \cdots a_{i_{k}} a_{i_{1}} \cdots a_{i_{k}} \cdots \cdots a_{i_{1}} \cdots a_{i_{k}}\right]$. Then, $\widetilde{\omega}\left(A_{1}, A_{2}, \ldots, A_{r}\right)=0$. According to Proposition 3, system (2) is zero output controllable.
Example 4. Consider the positive switched system composed of two subsystems with the following matrices:

$$
\begin{aligned}
& \left(A_{1}, B_{1}, C_{1}\right)=\left(\left[\begin{array}{llll}
0 & 1 & 0 & 0 \\
1 & 0 & 0 & 0 \\
0 & 0 & 1 & 0 \\
0 & 0 & 0 & 1
\end{array}\right],\left[\begin{array}{l}
1 \\
0 \\
0 \\
0
\end{array}\right],\left[\begin{array}{llll}
1 & 0 & 0 & 0 \\
1 & 0 & 0 & 0
\end{array}\right]\right), \\
& \left(A_{2}, B_{2}, C_{2}\right)=\left(\left[\begin{array}{llll}
1 & 0 & 1 & 1 \\
0 & 0 & 1 & 1 \\
0 & 0 & 0 & 1 \\
0 & 0 & 0 & 0
\end{array}\right],\left[\begin{array}{l}
0 \\
1 \\
0 \\
0
\end{array}\right],\left[\begin{array}{llll}
0 & 0 & 0 & 1 \\
0 & 0 & 0 & 1
\end{array}\right]\right) .
\end{aligned}
$$

By choosing $j=2$ and $\omega=a_{1} a_{2} \in \mathscr{A}^{*}$, we get $C_{2} \omega\left(A_{1}, A_{2}\right)=C_{2} A_{1} A_{2}=0$. Therefore, positive switched system (2) is zero output controllable. Also, the positive switched system (2) is zero output controllable, since there exists a word $\omega=a_{2} a_{1}$ such that $\left(\omega\left(A_{1}, A_{2}\right)\right)^{4}=0$, that is, $\omega\left(A_{1}, A_{2}\right)$ is nilpotent.

\section{Conclusions}

In this paper, we have addressed a number of issues related to the output reachability, output monomial reachability, and the zero output controllability properties of discretetime positive switched systems. By means of certain concepts borrowed from the algebra of noncommutative polynomials, we have been able to establish the necessary and sufficient conditions guaranteeing the output monomial reachability (Proposition 2) and the zero output controllability of discrete-time positive switched systems (Proposition 3). These conditions were then applied to numerical examples to illustrate their application and to support the theoretical results. The results discussed here will be of great value for our future work that will treat another class of positive systems.

\section{Data Availability}

No data were used to support this study.

\section{Conflicts of Interest}

The authors declare that they have no conflicts of interest regarding the publication of this paper.

\section{References}

[1] Y. Wang, C. Jiang, and Q. Wu, “Attitude tracking control for variable structure near space vehicles based on switched nonlinear systems," Chinese Journal of Aeronautics, vol. 26, no. 1, pp. 186-193, 2013.

[2] N. H. El-Farra, A. Gani, and P. D. Christofides, "A switched systems approach for the analysis and control of mode transitions in biological networks," in Proceedings of the 2005, American Control Conference, IEEE, Piscataway, NJ, USA, pp. 3247-3252, June 2005. 
[3] H. Torikai and T. Saito, "Synchronization of chaos and its itinerancy from a network by occasional linear connection," IEEE Transactions on Circuits and Systems I: Fundamental Theory and Applications, vol. 45, no. 4, pp. 464-472, 1998.

[4] K. Mitsubori and T. Saito, "Dependent switched capacitor chaos generator and its synchronization," IEEE Transactions on Circuits and Systems I: Fundamental Theory and Applications, vol. 44, no. 12, pp. 1122-1128, 1997.

[5] J. Klamka, A. Czornik, and M. Niezabitowski, "Stability and controllability of switched systems," Bulletin of the Polish Academy of Sciences: Technical Sciences, vol. 61, no. 3, pp. 547-555, 2013.

[6] D. Liberzon and A. S. Morse, "Basic problems in stability and design of switched systems," IEEE Control Systems Magazine, vol. 19, pp. 59-70, 1999.

[7] A. Babiarz, A. Czornik, J. Klamka, and M. Niezabitowski, Controllability of Discrete-Time Linear Switched Systems with Constrains on Switching Signal, Springer International Publishing, Switzerland, 2015.

[8] A. Babiarz and A. Czarnik, "Controllability of switched discrete-time fractional order systems with constrained switching sequence," in Proceedings of the 24th International Conference on Methods and Models in Automation and Robotics (MMAR), Międzyzdroje, Poland, August 2019.

[9] S. S. Ge, Z. Zhendong Sun, and T. H. Lee, "Reachability and controllability of switched linear discrete-time systems," IEEE Transactions on Automatic Control, vol. 46, no. 9, pp. 14371441, 2001.

[10] J. Klamka and M. Niezabitowski, "Controllability of switched linear dynamical systems," in Proceedings of the 18th International Conference on Methods and Models in Automation and Robotics, MMAR, 2013, pp. 464-467, Miedzyzdroje, Poland, August 2013.

[11] G. Ma and P. R. Pagilla, "Preview control of switched systems," in Proceedings of the 2019 IEEE/ASME International Conference on Advanced Intelligent Mechatronics (AIM), Hong Kong, China, July 2019.

[12] L. Tian, Y. Guan, and L. Wang, "Controllability and observability of switched multi-agent systems," International Journal of Control, vol. 92, no. 8, pp. 1742-1752, 2019.

[13] J. Zhang and T. Raïssi, "Saturation control of switched nonlinear systems," Nonlinear Analysis: Hybrid Systems, vol. 32, pp. 320-336, 2019.

[14] A. Babiarz, A. Czornik, and M. Niezabitowski, "Output controllability of the discrete-time linear switched systems," Nonlinear Analysis: Hybrid Systems, vol. 21, pp. 1-10, 2016.

[15] S. P. Meyn, Control Techniques for Complex Networks, Cambridge University Press, Cambridge, NY, USA, 2008.

[16] E. Seneta, Non-Negative Matrices and Markov Chains, Springer, Heidelberg, Germany, 2nd edition, 1981.

[17] A. K. Adebekun and F. J. Schork, "On the stability of some chemical processes via the theory of positive systems," Chemical Engineering Communications, vol. 69, no. 1, pp. 43-51, 1988.

[18] R. Shorten, F. Wirth, and D. Leith, "A positive systems model of tcp-like congestion control: asymptotic results," IEEE/ACM Transactions on Networking, vol. 14, no. 3, pp. 616-629, 2006.

[19] T. Kaczorek, "Realization problem for a class of positive continuous-time systems with delays," International Journal of Applied Mathematics and Computer Science, vol. 15, pp. 447-453, 2005.

[20] L. Farina and S. Rinaldi, Positive Linear Systems: Theory and Applications, John Wiley \& Sons, Hoboken, NJ, USA, 2011.
[21] I. Zorzan, "An introduction to positive switched systems and their application to HIV treatment modelling," University of Padua, Department of Information Engineering, Padua, Italy, 2014.

[22] E. Hernandez-Vargas, R. Middleton, and P. Colaneri, “Optimal and MPC switching strategies for mitigating viral mutation and escape," in Proceedings of the 2011 IFAC World Congress, Milano, Italy, September 2011.

[23] A. Jadbabaie, J. Lin, and A. Morse, "Coordination of groups of mobile autonomous agents using nearest neighbor rules," in Proceedings 41st IEEE Conference on Decision and Control, Las Vegas, NV, USA, December 2002.

[24] E. Fornasini and M. E. Valcher, "Stability and stabilizability criteria for discrete-time positive switched systems," IEEE Transactions on Automatic Control, vol. 57, no. 5, pp. 12081221, 2012.

[25] P. Santesso and M. E. Valcher, "Monomial reachability and zero controllability of discrete-time positive switched systems," Systems \& Control Letters, vol. 57, no. 4, pp. 340-347, 2008.

[26] J. Zhang, R. Zhang, X. Cai, and X. Jia, "A novel approach to control synthesis of positive switched systems," IET Control Theory \& Applications, vol. 11, no. 18, pp. 3396-3403, 2017. 